\title{
Reported measures of environmental tobacco smoke exposure: trials and tribulations
}

\author{
Melbourne F Hovell, Joy M Zakarian, Dennis R Wahlgren, Georg E Matt, \\ Karen M Emmons
}

Center for Behavioral Epidemiology and Community Health, Graduate School of Public Health, San

Diego State University, San Diego, California, USA

M F Hovell

J M Zakarian

D R Wahlgren

Department of Psychology, San Diego State University G E Matt

School of Public Health, Harvard University, Dana Farber Cancer Center, Boston,

Massachusetts, USA

K M Emmons

Correspondence to: Melbourne F Hovell, PhD, MPH, C-BEACH, 9245 Sky Park Court, Suite 230, San Diego, CA 92123, USA behepi@rohan.sdsu.edu

Received 6 December 1999 and in revised form

2000

Accepted 10 March 2000

\begin{abstract}
Objective-This report extends previous summaries of reported environmental tobacco smoke (ETS) exposure measures, reviews the empirical evidence of their validity for children's exposure, and discusses future research.

Data sources-Studies were identified by computer search and from the authors' research.

Study selection-Studies were selected for inclusion of nicotine and/or cotinine and quantitative reported measures of ETS exposure.

Data synthesis-Five studies found significant associations between reported quantitative exposure of children to ETS and either environmental nicotine or urine cotinine assays. Correlation coefficients between parent reports and nicotine ranged from 0.22 to 0.75 . Coefficients for cotinine ranged from $\mathbf{0 . 2 8}$ to $\mathbf{0 . 7 1}$. Correlations increased over time and were stronger for parents' reports of their own smoking as a source of children's exposure than for reports of exposure from others.

Conclusions-Empirical studies show general concordance of reported and either environmental or biological measures of ETS exposure. Relationships were moderate, and suggest sufficient validity to be employed in research and service programs. Future studies need to identify the differences in types of reported or objective measures, population characteristics, etc, contributing to observed variability in order to understand better the conditions under which more valid reported ETS exposure and other measures can be obtained. Reported and either environmental or biological measures should be used in combination, and existing measures should be directed to interventions that may reduce ETS exposure among children.

(Tobacco Control 2000;9(Suppl III):iii22-iii28)
\end{abstract}

Keywords: environmental tobacco smoke; children; nicotine; cotinine

Smoking is a leading cause of preventable morbidity/mortality. ${ }^{1}$ Environmental tobacco smoke (ETS) exposure is associated with lung cancer $^{2}$ and is classified as a carcinogen. ${ }^{3}$ ETS exposure in children is linked to respiratory infections, ear disease, asthma, and sudden death. ${ }^{3-9}$ ETS exposure has been estimated as the third leading preventable cause of death. ${ }^{10}$

Most smokers are of child bearing age and as many as $50 \%$ of children may be exposed to ETS. ${ }^{81-17}$ Research is imperative to determine exposure levels, disease risks, means of reducing ETS exposure, and consequential morbidity/mortality. However, such research is dependent on accurate measures. Summaries ${ }^{17} 18$ of common measures of ETS exposure, sources of error, and evidence for validity have outlined the value of questionnaire measures. However, most questionnaires have emphasised categorical and "indirect" measures of ETS, such as the smoking status (that is, yes/no) of parents in a home. ${ }^{17}{ }^{18}$ This review extends previous summaries to determine the validity of quantitative reported measures of children's ETS exposure and discusses their use in future studies.

Data sources and study selection

Studies were identified by computer search (Medline, PsycInfo) and from the authors' previous research. Studies were selected for inclusion of nicotine and/or cotinine and quantitative reported measures of ETS exposure. Five studies found significant associations between reported quantitative exposure of children to ETS and either environmental nicotine or urine cotinine assays, and results were quantitatively combined into summary measures.

\section{Research purposes}

Many studies are aimed at understanding disease/exposure relationships attributable to ETS exposure; others the behaviour that may result in exposure in specific microenvironments (for example, home). Different research purposes may require different measures, with varying levels of accuracy. To understand ETS related behaviour, information about social contexts that influence such behaviour is required. Even when environmental (for example, nicotine dosimeters) or biomarkers (for example, cotinine assays) are employed, complete interpretation of these measures has depended on reported information about context and sources of possible contamination/ confounding. Thus, even studies relying predominantly on environmental or biological measures may require accurate reported measures as well. 
Common measures of ETS exposure

ETS EXPOSURE MEASURES

ETS exposure is typically measured by report, or environmental nicotine or its metabolite cotinine, or by respirable suspended particles (RSP). ${ }^{18-22}$ Reported measures, nicotine and cotinine assays are specific to tobacco. ${ }^{23}$ Reported measures have ranged from living in a home with a smoker (a categorical and indirect estimate of ETS exposure) to detailed interviews and diary measures that include quantitative estimates of exposure ${ }^{17}$ Estimates of particle exposure are not specific to ETS, but may be important in order to assess potentially toxic exposure to particles not completely assessed by reported measures, nicotine or cotinine assays. ${ }^{22}$

WHAT IS AND IS NOT MEASURED?

Nicotine and cotinine provide estimates of exposure to an addictive constituent of ETS (nicotine), but are surrogate markers of other toxins produced by the burning of tobacco such as benzene, formaldehyde, etc. ${ }^{18}$ However, because nicotine and cotinine assays are highly correlated with most constituents of ETS, they can serve as measures for the combined but not separate toxic components. ${ }^{23}$ RSP measures of ETS are non-specific and may inadvertently include fireplace, cooking, and other sources of small particles of the size likely from ETS. However, because small particles can convey risk of disease owing to their size, and since the chemical makeup of particles can be another source of toxic exposure (including nicotine), particle measures as well as vapour phase estimates of ETS exposure may be important for complete estimates of ETS exposure or disease risk.

Nicotine and RSP assay rarely take into account ventilation, temperature, degree of dispersion, and other air physics that may influence the amount of smoke to which one is exposed. Lung size, frequency/depth of breathing, and metabolism (for example, speed of excretion as nicotine) may affect degree of intake/excretion of tobacco smoke toxins and hence the level of "internal" exposure. Biomarkers may vary (and consequentially the validity may vary) within and across individuals because of differences in metabolism. For instance, the half life for cotinine is substantially longer for infants and young children than adults (for example, 160 hours $v$ 24-40 hours, respectively). ${ }^{18}$ Within and between person variability complicate the interpretation of these measures. Saliva, blood, or urine samples yield related but not identical concentrations of cotinine, further complicating comparison across studies. Cotinine assays procedures and results also differ across studies, ${ }^{24}$ and laboratory tests vary in their sensitivity/specificity. For instance, common methods of analysing cotinine are radioimmunoassay (RIA), enzyme linked immunosorbent assay, and gas or liquid chromatography. For discriminating smokers from non-smokers, these have been accurate. However, chromatography has greater sensitivity, ${ }^{23}$ which may be necessary for detecting low level ETS exposure among children.

Estimates of ETS exposure based on nicotine and cotinine concentrations in body fluids may be confounded by food (for example, tomatoes) or from dust that is ingested or breathed. ${ }^{23}{ }^{25}$ Though only low doses are likely from such sources, cumulative exposure could confound estimates of ETS, especially in children exposed to a low level of ETS. Since nicotine/cotinine from foods are not markers for other toxic agents in tobacco smoke, nicotine and cotinine concentrations in body fluids to which food sources contributed may confound risk analyses. ${ }^{26}$ Though Pirkle and colleagues ${ }^{13}$ provide evidence of very small effects from diet, this issue has not been studied sufficiently to rule out sources of variance that could confuse estimates for special populations such as children.

Nicotine ingested or inhaled on dust particles (and subsequent cotinine metabolites of nicotine from dust) may reflect previous smoking in the environment, but not recent ETS exposure, or estimates of ETS exposure may be comprised of both current and past sources of nicotine ${ }^{27}$ in the same environment. Benowitz $^{23}$ estimates that this error might account for $0.1-0.3 \mathrm{ng} / \mathrm{ml}$ of cotinine and suggests this level of "error" is unlikely to compromise estimates of ETS. However, this source of variance has not been fully explored. Infants and children exposed to dust, and who ingest or are in close physical contact with nicotine contaminated objects (for example, cigarette butts), might have exposures substantially higher than would be the case from ETS exposure alone. While cotinine measures might truly reflect exposure to nicotine, they might not reflect sources limited to recent ETS exposure. When ETS exposure is defined as behaviour (that is, smoking in the presence of another person), cotinine measures may be less than ideal indicators of behavioural practices and these sources of "error" could reduce the correspondence between reported exposure and estimates based on a biomarker.

Measures of nicotine or cotinine are limited indicators of behaviour. Air sampling may provide information about the amount of smoke in a room, but not the presence of a would-be exposed person. Biological samples, assuming no confounding from sources other than cigarette smoke, might provide evidence of exposure, but not from whom, the timing, or other conditions. Smoking rates may not reflect the timing of exposure either. Emerson and colleagues found that while parents did not smoke much while driving, the car was one of the greatest sources of children's ETS exposure. ${ }^{28}$ Reported measures of exposure context remain important.

\section{REPORTED MEASURES OF EXPOSURE}

Reported measures raise numerous concerns about validity, especially false reports. Parents may over or under report ETS exposure (in order to avoid criticism, for example). Regardless of the motivation, the fact that reports can be falsified seems to set them apart from all 
other measures. Dosimeters may be "falsified" by smoking in a different room or tampering with the instruments, urine samples can be altered by not smoking for a few days before the sample or by substituting non-exposed persons' urine. However, risks of falsified assays are generally considered smaller than falsified reports. This risk of error justifies caution in the use or interpretation of reported measures; it does not justify assumptions of less accuracy than alternate measures.

Even if honest, reported measures may not be sensitive to "invisible" exposure. A child may be exposed by air conditioning, with no one smoking in the room and no smell or smoke visible. Parents may not report ETS exposure accurately if they are not present when the child is exposed, as when visiting a friend's home. Failure to take into account exposure when not observing the child can result in underestimates. Guessing about sources of exposure known to be likely but not directly observed, such as that from a routine caretaker who smokes, may result in over- or underestimates of ETS exposure. Matt and colleagues $^{21}$ showed that such estimates tended to overestimate ETS exposure.

\section{ATTENTION AND CUES}

Attention and memory can limit reports of ETS exposure. ${ }^{29}$ Most reported measures are based on recall. To recall children's exposure, it must have been discriminated at the time of occurrence. This may be influenced by competing events, such as watching TV, conversation, etc. Memory also may be influenced by conditions taking place when completing a questionnaire or interview. Distractions can limit reports and interviewer prompts may enhance recall; the type of item and their number may increase or decrease precision and accuracy. Thus, the validity and completeness of reported measures depend on context, skill, and attention and might be enhanced by carefully designed items. Most questionnaire measures of ETS have relied on categorical assessments (for example, live with a smoker), or "indirect" measures such as the number of cigarettes a parent smokes. ${ }^{17}$ These types of measures provide limited information about the degree of exposure and might imply exposure when almost none actually takes place. This can happen, for instance, when a smoking parent never smokes around his or her child.

\section{REACTIVITY}

For all measures of ETS exposure, concerns of reactivity are warranted. Dosimeters in the home, asking parents to collect biological samples, or asking parents to report their child's ETS exposure, may change "exposure behaviour". This has been seen in the study by Hovell and colleagues, ${ }^{30}$ with all groups reporting less exposure over repeated baseline measures. These results suggest that parents change their "exposure behaviour" in response to measures alone. For studies attempting to determine the usual/typical level of exposure, this type of reactivity will lead to lower than true estimates of level and change. Remarkably, this type of reactivity is not discussed in the few extensive reviews of ETS measures. ${ }^{17} 1823$

Placing nicotine or particle dosimeters in the home, obtaining saliva, blood or urine samples for cotinine assays, or repeatedly asking a parent about their child's exposure to ETS may lead to changes in reported information. Sensitising parents to ETS by use of any or all of these measures may increase their ability to detect ETS exposure, especially over time. This could result in increasingly more valid reported measures of ETS exposure, such as seen by Emerson and colleagues ${ }^{31}$ and Matt and associates. $^{29}$ While not yet reported in the literature, it also can be expected that information about the context of ETS exposure - such as the timing, place, activities involved-will be changed by the parents' sensitivity owing to the invasive nature of measures. This could lead to more accurate information over time about the context of exposure. Alternatively, such sensitivity could lead to parents reporting less accurately, perhaps to avoid implied criticism for their child's ETS exposure. These sources of bias have not been studied adequately. If over or under reporting is different for participants in varying experimental conditions or exposure groups, this type of bias could cause systematic classification error as well as overor underestimates of exposure magnitude. Matt and colleagues have shown that validity coefficients differ by experimental groups. ${ }^{29}$

\section{Validity of reported ETS exposure}

Investigators have reviewed problems with environmental, biological, and reported measures of ETS exposure. ${ }^{182023-25}$ These reviews and the discussion above outline numerous sources of possible variance in all measures. These many sources of variance complicate the interpretation of cross measure correspondence as evidence of validity. However, such correspondence remains the best means of estimating validity for any available measure. While Jarvis ${ }^{17}$ has concluded that cotinine is the "gold standard" by which reported measures may be validated, the discussion above provides substantial reason to consider this premature. Rather, cotinine is simply one measure of many (for example, nicotine, particle exposure, etc) that can contribute to the test of validity of reported measures.

ADULT REPORTED ETS EXPOSURE

Coughlin and associates had 19 non-smoking adults wear dosimeters near the breathing zone and reported the number of cigarettes smoked in their presence during the time period they carried the dosimeter. ${ }^{32}$ Nicotine was assessed using gas chromatography. Across reported exposure scales, correlation coefficients ranged from 0.72 to 0.95 . This suggested a high level of validity, but only under extremely limited conditions unlikely for most field uses. Emmons and colleagues obtained saliva cotinine determined by gas chromatography, questionnaire, and diary reported ETS 
exposure for 186 non-smoking adults. ${ }^{33}$ Coefficients ranged from 0.22 to 0.42 . These studies show considerable variability in relationships and may not represent findings true for children.

\section{SMOKING RATES AS PROXY MEASURES OF ETS} EXPOSURE

Greenberg and associates, using RIA techniques for 44 mother/child pairs, examined the relationship between mothers' self reported smoking rates (an indirect estimate of ETS exposure) and children's nicotine and cotinine assays. ${ }^{34}$ The strongest $(r=0.67)$ was for urine cotinine. Parent smoking rates also were employed by other investigators as indicators of ETS exposure. Bahceciler and colleagues studied 77 asthmatic children, using RIA assays of urine samples, and showed a moderate correlation $(r \sim 0.52)$ with parent smoking rates. ${ }^{35}$ Marbury and associates studied 48 infants, using RIA urine cotinine assays and nicotine dosimeters. ${ }^{36}$ Strong relationships (for example, Spearman correlations of 0.80 to 0.91 ) were reported for cotinine and nicotine and for smoking rates and these two measures. Crawford and colleagues studied 87 minority preschool children and reported significant associations with mothers' smoking rate. ${ }^{37}$ Nafstad and associates reported Spearman rank correlations for hair nicotine (0.64) and urine cotinine $(0.50)$ with reported parent smoking rates. ${ }^{38}$ The relationship between nicotine and cotinine was essentially the same (0.56). Oddoze and colleagues also used smoking rates as proxy measures of ETS exposure for 90 asthmatic children. ${ }^{39}$ They reported highly significant Spearman correlations with cotinine. The consistency among studies for parents' smoking rate as an indirect measure of the child's exposure is encouraging for estimates of prevalence. However, these relationships should be confirmed with direct measures of ETS exposure, because ETS exposure can change without parent smoking rates changing. Thus, for experimental evaluation of interventions, indirect measures may be questionable.

\section{QUANTITATIVE REPORTED ETS EXPOSURE}

MEASURES

Jaakkola and Jaakkola ${ }^{18}$ pointed out the importance of a quantitative measure of ETS exposure. Few studies to date have provided estimates of the number of cigarettes (or alternate quantitative estimates) to which a child may be exposed and compared this estimate of ETS exposure to environmental or biological markers. Ogborn and colleagues investigated an ordinal scale of degree of reported exposure and urine cotinine measured by RIA for 58 asthmatic children. ${ }^{40}$ Spearman rank correlations ranged from 0.35 to 0.41 among acute patient visits and from 0.44 to 0.48 among well patient visits. Questions about exposure were not specific to the parents as sources of exposure, and this may have decreased under reporting error.

Emerson and colleagues examined parent reported number of cigarettes smoked while the child was present as an estimate of ETS exposure, and air nicotine estimates for 91 asthmatic children. ${ }^{31}$ Nicotine was measured by gas chromatography. Tau $\beta$ coefficients between nicotine and reported measures ranged from 0.22 to 0.35 for interviews, from 0.27 to 0.30 for diaries, and from 0.29 to 0.47 for cigarette butts. Most relationships increased over time.

Fried and colleagues ${ }^{41}$ asked parents of 109 children to estimate the number of hours of exposure. Correlation coefficients comparing these estimates with cotinine ranged from 0.66 to 0.71 depending upon adjustment for creatinine and/or age and sex.

Matt and colleagues ${ }^{21}$ examined mother reported measures of the number of cigarettes smoked while the child was present and urine cotinine (using isotope dilution liquid chromatography tandem mass spectrometry (ID-LC/ $\mathrm{MC} / \mathrm{MS}$ ) with a detection concentration of $0.05 \mathrm{ng} / \mathrm{ml})^{19}$ for 16 infants and 20 children from 3 to 8 years old. Pearson correlation coefficients ranged from 0.28 to 0.63 for different measures and times.

Matt and colleagues ${ }^{29}$ also investigated parent reported ETS exposure (that is, number of cigarettes smoked in the presence of the child) and chromatograph measures of nicotine $(n=68)$ and cotinine $(n=141$ well children). Pearson correlation coefficients for nicotine and different types of reported measures ranged from 0.69 to 0.75 for an experimental group and from 0.35 to 0.48 for controls. Relationships for cotinine ranged from 0.52 to 0.62 in the experimental group and 0.35 to 0.66 for controls. Cotinine relationships increased over time. The relationship between nicotine and cotinine yielded correlations of 0.74 for the experimental group and 0.48 for controls, essentially the same size relationship as found for reported measures and each of these measures. Relationships were strongest among experimental group families, suggesting that counselling to avoid ETS exposure may increase parents' reporting skills. The lower validity coefficients for control group families compared to those in an intervention designed to decrease exposure, raises the possibility of "differential misclassification error". ${ }^{18}$

\section{OVERALL VALIDITY: DATA SYNTHESIS}

Overall, the relationships between environmental and biological indicators of ETS exposure corresponded moderately with quantitative reported measures. We combined the correlations from the five independent studies of reported quantitative children's ETS exposure $^{2129314041}$ by determining the weighted mean of the z'-transformed correlations for lower and upper ends of the range. ${ }^{42}$ The weight associated with each correlation is given by the inverse of the sampling variance of the $\mathrm{Z}$ '-transformed correlation. $\mathrm{Z}$ tests were conducted to test the null hypothesis that the population parameter rho is zero. The mean lower boundary estimate based on the lower level coefficients reported was 0.43 (95\% confidence interval (CI) 0.33 to $0.49 ; z=9.0$, 
$\mathrm{p}<0.0001)$. At worst, reported exposure and environmental and biological measures share about $18.5 \%$ of their variance. These correlations were heterogeneous $\left(\chi^{2}(4)=19.1\right.$, $\mathrm{p}<0.001)$, and suggest that correlations vary more than expected because of sampling error. When the upper boundary estimates were used, the mean correlation was $0.63(95 \%$ CI 0.57 to $0.69 ; \mathrm{z}=15.3, \mathrm{p}<.0001)$. At best, reported exposure and biological measures share up to $40 \%$ of their variance. These correlations were also heterogeneous $\left(\chi^{2}(4)=24.6\right.$, $\mathrm{p}<0.001)$. Because findings from these studies are not homogeneous, sources of variance in validity coefficients may include differences in populations represented by the various samples as well as sources of error among measures. To understand better the validity of reported measures of ETS exposure, it will be important to identify the specific study characteristics (for example, urine specimen in the field, type of laboratory analyses, type of parent reports, participants) that contributed to the observed heterogeneity of validity coefficients.

The consistency in direction of these associations across independent studies is reassuring and suggests that reported measures can be satisfactory indicators of exposure. This conclusion is bolstered by the observation that relationships between reported measures and biological indicators are about the same magnitude as the relationship (for example, $r=0.68$ ) between biological and environmental measures. ${ }^{29} 43$

REACTIVITY

The conclusion that reported measures are valid is qualified by the fact that correspondence between reported ETS exposure measures and environmental or biological markers have been under conditions where respondents were aware of possible confirmation of their reports. It remains to be determined how accurate reported measures might be if used without concurrent environmental and/or biological assays.

NO “GOLD STANDARD"

While there is general correspondence between parent reported exposure and children's cotinine, and environmental nicotine, these measures are far from providing identical information. The failure to obtain greater correspondence is likely due to error in all measures and because these measures do not assess the same things. Future research should address inter-individual differences affecting the air physics taking into account toxic concentrations, type of cigarettes, ventilation, dispersion, and other factors that may influence the degree of exposure in microenvironments. Similarly, greater understanding of breathing patterns, lung function and size, and metabolism (by age, sex, and health conditions) are needed to understand the sources of variance in biomarkers. ${ }^{18}$ Reported measures warrant investigation as well. Matt and colleagues have shown that "fuzzy set" measures, reporting high and low ranges of exposure, can account for an additional 8.7\% of variance in babies' cotinine compared to point estimates alone. ${ }^{29}$ This suggests that the type of question and computed estimate of exposure may influence validity. The increase in coefficients over time suggests that respondents learn to report ETS exposure more accurately with experience. ${ }^{29}{ }^{31}$ While this is problematic when comparing groups of unequal experience, it suggests that research should determine the experience that equips participants with accurate observation and reporting skills.

ACUTE VERSUS CUMULATIVE EXPOSURE

Reported, environmental, or biological measures of ETS exposure provide information about acute exposure. Cumulative exposure estimates require continuous or frequently repeated measures. Hair samples offer "longer term" estimates (that is, 1-2 months). ${ }^{44} 45$ However, there are many potential sources of error and few studies of validity. Hair samples offer promising measures of nicotine and cotinine, and warrant more study. Thus, common measures serve well as estimates for settings that do not change often and as satisfactory measures of interventions for reducing ETS exposure in the short term. Studies of cumulative toxic exposure or long term behaviour patterns require more frequent use of a common measure or new continuous measures.

\section{Real time and continuous measures}

IMMEDIATE VERSUS DELAYED MEASURES

Environmental and biological assays are collected intermittently and returned to laboratories for delayed analyses. Reported measures are usually taken intermittently and may take weeks to summarise. The failure to obtain real time and continuous (or close approximation) measures of ETS exposure limits our understanding of true exposure levels, cumulative exposure levels, and variability over time (that is, exposure-time profile). Current measures also do not often include estimates of sources of variability over time. Without more information about variability over time and associated events that might account for such variability, efforts to control ETS exposure will be compromised.

\section{FEEDBACK}

A profound means of influencing human behaviour is immediate feedback. Real time measures of ETS exposure, as feedback, could influence ETS exposure practices, perhaps yielding greater effects than already seen in parents reactivity to discretely sampled measures. ${ }^{30}$ Most of the technology needed to develop fine particle dosimeters that can be convenient, nearly continuous, and from which immediate feedback can be derived is already available. $^{46-49}$ To our knowledge no real time measures of biomarkers are available, but by analogy it should be possible to develop tools similar to that used by diabetic patients to obtain frequent blood glucose samples. Automated and digital video recording equipment is now available and could serve as 
another means of recording visible tobacco smoke and persons present. Miniature computer systems are available by which recorded data could be summarised automatically, providing more informative sources of feedback (for example, trends over time). Research and development should be directed toward more accurate, real time, and continuous indicators of exposure. Such measures may enhance our understanding of disease associations and enhance investigators' and parents' ability to reduce children's ETS exposure beyond what may be possible from seeing or smelling tobacco smoke.

\section{Behavioural interventions}

Efficacious counselling to control ETS exposure has been dependent on "clinical measures" as a basis for directing behaviour change. ${ }^{30} 50$ The fact that these "clinical measures" have been sufficiently accurate to make possible efficacious counselling is remarkable. Improved measures might increase the power of minimal interventions previously found ineffective. ${ }^{51-53}$ Clinicians are in a unique position to employ both biomarkers (for example, cotinine) and interview measures repeatedly over office visits. If results were used for both advice and feedback, the efficacy of clinician counselling might increase. Even though ideal measures are not yet available, existing combinations of sensitive cotinine and interview measures could be used by clinicians. These might be sufficient to make repeated clinician advice efficacious. While new measures undergo development, available quantitative reported measures along with either environmental and/or biomarkers should be employed as tools for estimating and controlling exposure.

\section{Conclusions and recommendations}

Commonly employed measures of ETS exposure include nicotine, cotinine, and reports. Each assesses different things, none of which serve as a complete or completely valid measure of ETS exposure. All currently employed measures assess relatively acute exposure. Cumulative exposure requires development of new measures or frequent use of existing measures longitudinally. Research is needed to understand better the sources of error for all available measures and from which decreased error can be engineered. Among needed studies, development of real time and continuous measures would enhance epidemiological and intervention studies for control of ETS exposure.

Current quantitative reported measures of ETS exposure offer promise as sufficiently valid to be used in community studies and health services. However, reported measures should be elicited in the context of concurrent environmental or biological indicators in order to both sensitise participants to possible confirmation of their reports and provide more than one indicator of exposure. Though many trials and tribulations remain for the use of reported and other measures of ETS exposure, the validity of quantitative reported measures meet the validity standards now considered satisfactory for both environmental and biological measures of ETS. This suggests that each type of measure is about equally valid (or equally incompletely valid). It also suggests that current measures, if used in combination, meet the standards necessary for larger scale epidemiological and clinical trials from which it may be possible to learn how to control children's ETS exposure at home.

Preparation of this report was supported by grants 027946 SFP and 027940 SFP from the Robert Wood Johnson Foundation Smoke-Free Families Program awarded to Drs Hovell and Emmons. The authors wish to thank $\mathrm{H}$ Pennington Whiteside, Jr, MSPH, Deputy Director of the Smoke-free Families Program, for his assistance.

1 US Department of Health and Human Services. Reducing the health consequences of smoking: 25 years of progress. A report of the Surgeon General, 1989. Rockville, Maryland: Public Health Service, Centers for Disease Control, Office on Smoking and Health, 1989. (DHHS Publication No (CDC) 89-8411.)

2 US Department of Health and Human Services. The health consequences of involuntary smoking. A report of the Surgeon General, 1986. Rockville, Maryland: Public Health Service, Centers for Disease Control, 1986. (DHHS Publication No (CDC) 87-8398.)

3 US Environmental Protection Agency. Respiratory health effects of passive smoking: lung cancer and other disorders. Assessment, 1992. (Publication No EPA/600/6-90/006F.)

4 California Environmental Protection Agency. Health effects of exposure to environmental tobacco smoke. Sacramento, California: California Environmental Protection Agency, Office of Environmental Health Hazard Assessment, September 1997.

5 Australian National Health and Medical Research Council. The health effects of passive smoking. Australian Government Publishing Service, ISBN 0-642-27270-0, 1997.

6 Scientific Committee on Tobacco and Health. Report of the scientific committee on tobacco and health, 1998. http:// www.official-documents.co.uk/document/doh/tobacco/ contents.htm.

7 Cook DG, Strachan DP. Summary of effects of parental smoking on the respiratory health of children and implications for research. Thorax 1999;54:357-66.

8 World Health Organization. International consultation on environmental tobacco smoke and child health: consultation report. Geneva: WHO, 1999. http://www.who.int/toh/TFI/ consult.htm.

9 Wahlgren DR, Hovell MF, Meltzer EO, Meltzer SB. Involuntary smoking and asthma. Curr Opin Pulmonary Med 2000;6:31-6.

10 Glantz SA, Parmley WW. Passive smoking and heart disease: epidemiology, physiology, and biochemistry. Circulation 1991;83:1-12.

11 US Department of Health and Human Services. Preventing tobacco use among young people. A report of the Surgeon General, 1994. Atlanta, Georgia: Public Health Service, Centers for Disease Control and Prevention, Office on Smoking and Health, 1994. (US Government Printing Office Publi-001-00491-0.)

12 Pierce JP, Gilpin E. How long will today's new adolescent smoker be addicted to cigarettes? Am $\mathcal{f}$ Public Health 1996;86:253-6

13 Pirkle JL, Flegal KM, Bernert JT, et al. Exposure of the U.S. population to environmental tobacco smoke: The third National Health and Nutrition Examination Survey, 1988 to 1991. FAMA 1996;275:1233-40.

14 Gergen PJ, Fowler JA, Maurer KR, et al. The burden of environmental tobacco smoke exposure on the respiratory health of children 2 months through 5 years of age in the United States: Third National Health and Nutrition Examination Survey, 1988 to 1994. Pediatrics 1998; 101:E8.

15 Collaborative Group SIDRIA (Italian Studies on Respiratory Disorders in Childhood and the Environment). Parental smoking, asthma and wheezing in children and adolescents. Results of SIDRIA. Epidemiol Prev 1998; 22:146-54.

16 Lister SM, Jorm LR. Parental smoking and respiratory illnesses in Australian children aged $0-4$ years: ABS 1989-90 National Health Survey results. Aust NZ f Public Health 1998;22:781-6.

17 Jarvis MJ. Background paper: children's exposure to passive smoking: survey methodology and monitoring trends. International Consultation on Environmental Tobacco Smoke and Child Health. World Health Organization, 1999. http://www.who.int/toh/TFI/consult.htm.

18 Jaakkola MS, Jaakkola JJK. Assessment of exposure to environmental tobacco smoke. Eur Respir f 1997;10:2384-97.

19 Bernert JT, Turner WE, Pirkle JL, et al. Development and validation of sensitive method for determination of serum cotinine in smokers and nonsmokers by liquid chromatography/atmospheric pressure ionization tandem chromatography/atmospheric pressure ionization
mass spectrometry. Clin Chem 1997;43:2281-91. 
20 Hammond SK, Leaderer BP. A diffusion monitor to measure exposure to passive smoking. Env Sci Tech 1987, measure expc

21 Matt GE, Wahlgren DR, Hovell MF, et al. Measuring ETS exposure in infants and young children through urine cotinine and memory-based parental reports: empirical findings and discussion. Tobacco Control 1999;8:282-9.

22 Rickert WS. Background paper: Environmental tobacco smoke: properties, measurement techniques and applications. International Consultation on Environmental Tobacco Smoke and Child Health. World Health Organi-

23 Benowitz NL. Cotinine as a biomarker of environmenta tobacco smoke exposure. Epidemiol Rev 1996;18:88-204.

24 Idle J. Titrating exposure to tobacco smoke using cotinine-a minefiel

25 Davis RA, Stiles MF, deBethizy JD, et al. Dietary nicotine: a aris RA, Stiles MF, deBethizy JD, et al. Dietary nicotine: source of urinary cotinine. Fd Chem Toxic 1991;29:821-7.

26 Domino EF. Estimating exposure to environmental tobacco smoke. FAMA 1996;276:603.

27 Nelson PR, deBethizy JD, Davis RA, et al. Where there's smoke ...? Biases in the use of nicotine and cotinine as environmental tobacco smoke biomarkers. In: Proceeding of the 1991 Environmental Protection Agency/Air and Waste Management Association International Symposium: Measurement of toxic and related air pollutants. Pittsburgh, Pennsylvania: Air and Waste Management Association, 1991:449-54.

28 Emerson JA, Wahlgren DR, Hovell MF, et al. Parent smoking and asthmatic children's exposure patterns: a behavioral epidemiology study. Add Behav 1994;19:677-89.

29 Matt GE, Hovell MF, Zakarian JM, et al. Measuring second-hand smoke exposure in babies: reliability and validity of mother-reports in a sample of low-income families. Health Psych In press.

30 Hovell MF, Meltzer SB, Zakarian JM, et al. Reduction of environmental tobacco smoke exposure among asthmatic environmental tobacco smoke exposure among ast
children: a controlled trial. Chest 1994;106:440-6.

31 Emerson JA, Hovell MF, Meltzer SB, et al. The accuracy of environmental tobacco smoke exposure measures among asthmatic children. F Clin Epidemiol 1995;48:1251-9.

32 Coughlin J, Hammond SK, Gann PH. Development of epidemiologic tools for measuring environmental tobacco smoke exposure. Am f Epidemiol 1989;130:696-704

33 Emmons KM, Abrams DB, Marshall R, et al. An evaluation of the relationship between self-report and biochemical measures of environmental smoke exposure. Prev $\mathrm{Med}$ 1994;23:35-9.

34 Greenberg R, Haley N, Etzel R, et al. Measuring the exposure of infants to tobacco smoke: nicotine and cotinine in urine and saliva. N Engl f Med 1984:310:10758.

35 Bahceciler NN, Barlan IB, Nuhuglu Y, et al. Parental smoking behavior and the urinary cotinine levels of asthmatic ing behavior and the urinary coting

36 Marbury MC, Hammond SK, Haley NJ. Measuring exposure to environmental tobacco smoke in studies of acute health effects. Am $\mathcal{F}$ Epidemiol 1993;137:1089-97.
37 Crawford FG, Mayer J, Santella RM, et al. Biomarkers of environmental tobacco smoke in preschool children and

38 Nafstad P, Botten G, Hagen JA, et al. Comparison of three methods for estimating environmental tobacco smoke exposure among children aged between 12 and 36 months. Int $\mathcal{F}$ Epidemiol 1995;24:88-94.

39 Oddoze C, Dubus JC, Badier M, et al. Urinary cotinine and exposure to parental smoking in a population of children with asthma. Clin Chem 1999;45:505-9.

40 Ogborn CJ, Duggan AK, DeAngelis C. Urinary cotinine as a measure of passive smoke exposure in asthmatic children. Clin Pediatr 1994;33:220-6.

41 Fried PA, Perkins SL, Watkinson B, et al. Association between creatinine-adjusted and unadjusted urine cotinine values in children and the mother's report of exposure to environmental tobacco smoke. Clin Chem 1995;28:41520.

42 Shadish WR, Haddock CK. Combining estimates of effect size. In: Cooper H, Hedges LV, eds. The handbook of research synthesis. New York: Russell Sage Foundation, 1994: 261-81.

43 Henderson FW, Reid HF, Morris R, et al. Home air nicotine levels and urinary cotinine excretion in preschool children. Am Rev Respir Dis 1989;140:197-201.

44 Zahlsen K, Nilsen OG. Nicotine in hair of smokers and non-smokers: sampling procedure and gas chromatograph/mass spectrometric analysis. Pharmacol Toxicol 1994;75:143-9.

45 Kintz P. Gas chromatographic analysis of nicotine and cotinine in hair. 7 Chromatogr 1992;580:347-53.

46 Turner W, Spengler J, Dockery D, et al. Design and performance of a reliable personal monitoring system of respirable particulates. If Air Pollution Control Assoc 1979;29:747-8

47 Spengler J, Treltman R, Tosteson D, et al. Personal exposures to respirable particulates and implications for air pollution epidemiology. Env Sci Tech 1985;19:700-7.

48 Muramatsu M, Umemura S, Okada T, et al. Estimation of personal exposure to tobacco smoke with a newly developed nicotine personal monitor. Environ Res 1984; 35:218-27.

49 Brauer M, Hirtle RD, Hall AC, et al. Monitoring personal fine particle exposure with a particle counter. $\mathcal{F}$ Expo Anal Environ Epidemiol 1999;9:228-36.

50 Wahlgren DR, Hovell MF, Meltzer SB, et al. Reduction of environmental tobacco smoke exposure in asthmatic children: a two-year follow-up. Chest 1997;111:81-8.

51 Irvine L, Crombie I, Clark R, et al. Advising parents of asthmatic children on passive smoking: randomized controlled trial. BMF 1999;318:1456-9.

52 Woodward A, Owen N, Gurinovich N, et al. Trial of an intervention to reduce passive smoking in infancy. Pediatr Pulmonol 1987;3:173-8.

53 Chilmonczyk B, Palomaki G, Knight G, et al. An unsuccessful cotinine-assisted intervention strategy to reduce environmental tobacco smoke exposure during infancy. Am f Dis Child 1992;146:357-60.

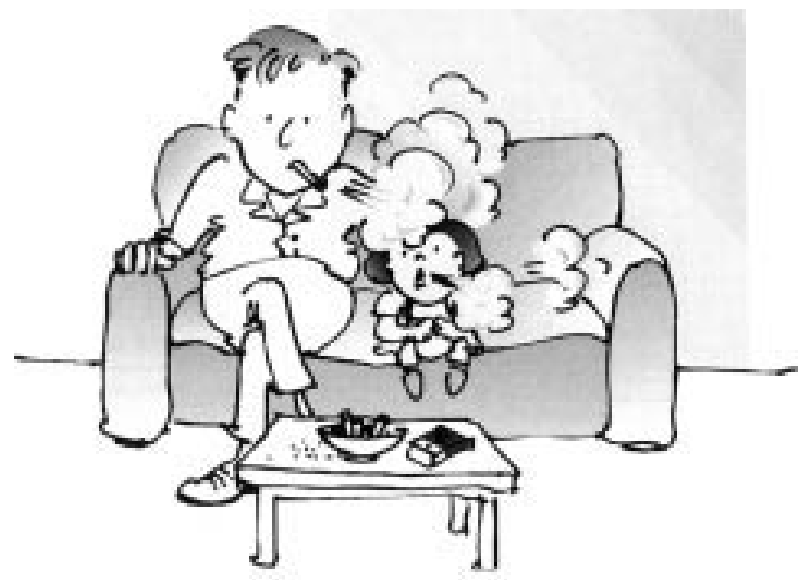

Contributed by Melanie Wakefield. 